\title{
Ocular Myositis Occurring with NOD2-associated Autoinflammatory Disease
}

QINGPING YAO, MD, PhD; PAUL RUGGIERI, MD; and CAREEN LOWDER, MD, Departments of Rheumatic and Immunologic Diseases, Neuroradiology, and Ophthalmology, Cole Eye Institute, Cleveland Clinic, Cleveland, Ohio, USA. Address correspondence to Dr. Q. Yao, Department of Rheumatic and Immunologic Diseases/A50, Cleveland Clinic, 9500 Euclid Avenue, Cleveland, Ohio 44195, USA. E-mail: yaoq@ccf.org. J Rheumatol 2013;40:1768-9; doi:10.3899/jrheum.130332

NOD2-associated autoinflammatory disease (NAID) is an emerging disease ${ }^{1}$. We report a patient with ocular myositis (OM) whose clinical phenotype was consistent with NAID.

A 34-year-old white woman developed eyelid swelling, pain on eye movement, and color vision loss 3 years ago. There was right eye pain with adduction and abduction with normal visual acuity and color vision on examination. A magnetic resonance imaging study revealed findings for
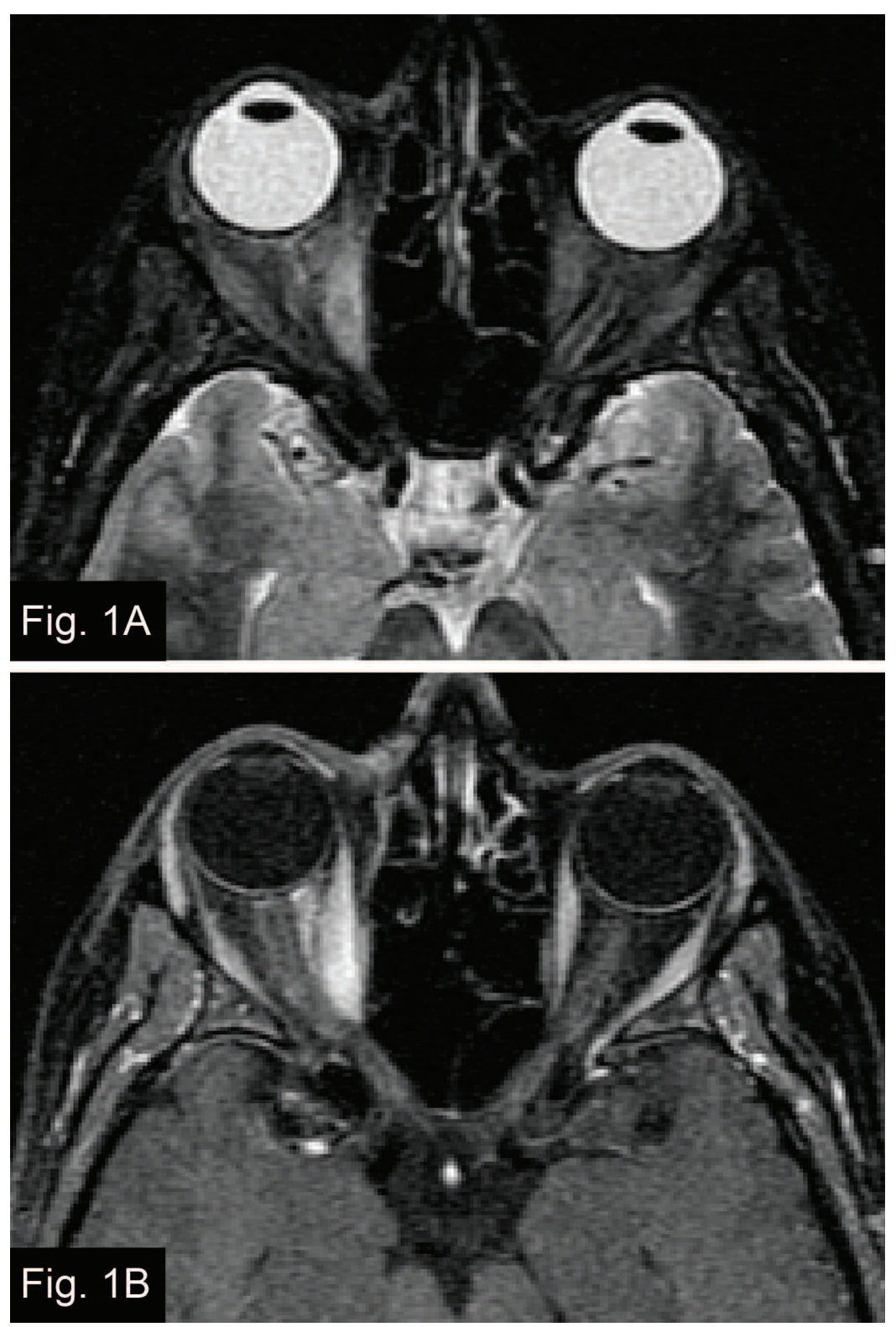

Figure 1. A. Fat-suppressed T2-weighted image of the orbits demonstrating focal fusiform enlargement and hyperintensity in the right medial rectus muscle with sparing of the tendonous insertions. B. Fat-suppressed gadolinium enhanced T1-weighted image with prominent enhancement of the involved portion of the right medial rectus muscle and mild ill-defined enhancement of the intraconal fat. 


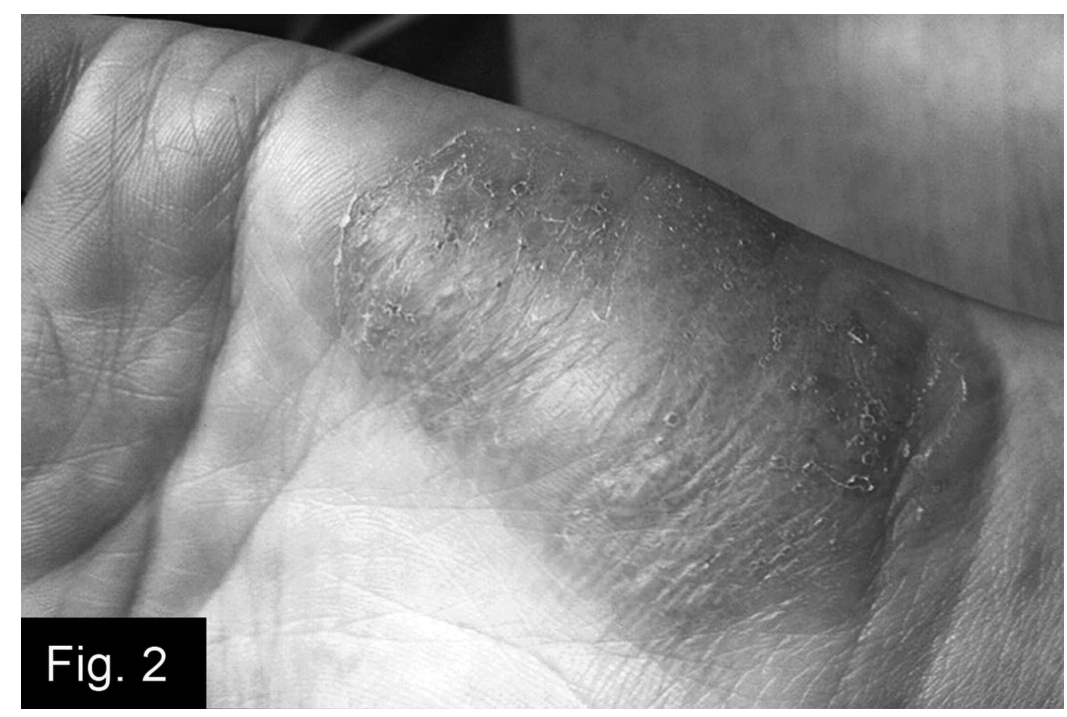

Figure 2. Palmar erythema at the hypothenar eminences with scattered pustules and scales.

OM (Figure 1A, 1B). Her OM gradually resolved with oral prednisone and radiation therapy. She developed red patches on the hypothenar eminences (Figure 2) and feet consistent with spongiotic dermatitis. She had inflammatory polyarthritis involving the proximal interphalangeal joints of the hands, wrists, shoulders, knees, and ankles for the past 6 years. She also had low-grade fever that recurred 3 to 4 times a year, with each episode lasting for 1 or 2 days. Other symptoms included fatigue, mild weight loss, night sweats, recurrent oral ulcers, and 2 episodes of genital vulvar ulcers, which resolved with sulfasalazine treatment. Laboratory evaluations, including serologic testing for systemic autoimmune diseases, were negative, as were HLA-B27 and B51. Genetic testing was positive for heterozygous R702W and IVS8+158 variants of NOD2.

The clinical phenotype of this patient is consistent with NAID. It is characterized by periodic fever, dermatitis, and polyarthritis. In addition, patients can also experience weight loss, and gastrointestinal and sicca-like symptoms.
NAID is linked to the NOD2 mutations IVS $8+158, \mathrm{R} 702 \mathrm{~W}$, and $\mathrm{R} 703 \mathrm{C}^{1-3}$. To our knowledge, this is the first report of OM with NOD2. There was no evidence of Crohn disease. NOD2 mutations have not been associated with Behçet disease $^{4}$.

\section{REFERENCES}

1. Yao Q, Su LC, Tomecki KJ, Zhou L, Jayakar B, Shen B. Dermatitis as a characteristic phenotype of a new autoinflammatory disease associated with NOD2 mutations. J Am Acad Dermatol 2013;68:624-31.

2. Yao Q, Zhou L, Cusumano P, Bose N, Piliang M, Jayakar B, et al. A new category of autoinflammatory disease associated with NOD2 gene mutations. Arthritis Res Ther 2011;13:R148.

3. Yao Q, Piliang M, Nicolacakis K, Arrossi A. Granulomatous pneumonitis associated with adult-onset Blau-like syndrome. Am J Respir Crit Care Med 2012;186:465-6.

4. Kappen JH, Wallace GR, Stolk L, Rivadeneira F, Uitterlinden AG, van Daele PL, et al. Low prevalence of NOD2 SNPs in Behcet's disease suggests protective association in Caucasians. Rheumatology 2009;48:1375-7. 